\title{
Review of: "Tobacco-containing product"
}

\author{
Clive Bates
}

Potential competing interests: The author(s) declared that no potential competing interests exist.

I have four points about the definition: "A product that contains or consists of tobacco and is used by people to ingest tobacco constituents."

1. "or consists of" is redundant.

2. "ingest" usually means to eat or absorb via the digestive tract and would be normally seen as different to absorption through inhalation "Absorb" would be better as it allows for absorption in the oral cavity, lungs, throat or by any route.

3. In the case of smoking, the user is not absorbing tobacco constituents but many de novo products of combustion that are not present in tobacco itself.

4. For clarity, the definition should address the confusion about extracts made from tobacco leaf (for example, extracts of nicotine or natural extracts of tobacco used in tobacco flavoured vaping products, cosmetics or soaps). It would be best if 'tobacco' was clarified to be restricted to parts of the tobacco plant in raw and cured form.

A suggested alternative definition:

"A product that contains whole tobacco in raw or cured form and is used by people to absorb tobacco constituents or products of tobacco combustion. It does not include products made with extracts from the tobacco plant." 